\title{
Common respiratory issues in ambulatory obstetrics.
}

\author{
Kelly M Orzechowski \\ Thomas Jefferson University \\ Richard C Miller \\ St. Barnabas Medical Center
}

Follow this and additional works at: https://jdc.jefferson.edu/obgynfp

Part of the Obstetrics and Gynecology Commons

Let us know how access to this document benefits you

\section{Recommended Citation}

Orzechowski, Kelly M and Miller, Richard C, "Common respiratory issues in ambulatory obstetrics." (2012). Department of Obstetrics and Gynecology Faculty Papers. Paper 26.

https://jdc.jefferson.edu/obgynfp/26

This Article is brought to you for free and open access by the Jefferson Digital Commons. The Jefferson Digital Commons is a service of Thomas Jefferson University's Center for Teaching and Learning (CTL). The Commons is a showcase for Jefferson books and journals, peer-reviewed scholarly publications, unique historical collections from the University archives, and teaching tools. The Jefferson Digital Commons allows researchers and interested readers anywhere in the world to learn about and keep up to date with Jefferson scholarship. This article has been accepted for inclusion in Department of Obstetrics and Gynecology Faculty Papers by an authorized administrator of the Jefferson Digital Commons. For more information, please contact: JeffersonDigitalCommons@jefferson.edu. 


\title{
As submitted to: \\ Clinical obstetrics and gynecology.
}

And later published as:

Common Respiratory Issues in Ambulatory Obstetrics

September 2012, Volume 55, Issue 3, pp. 798-809

DOI: $10.1097 / G R F .0 b 013 e 31825 c f d 17$.

\author{
Kelly M. Orzechowski, MD, MPH \\ Thomas Jefferson University Hospital \\ Division of Maternal Fetal Medicine \\ Department of Obstetrics \& Gynecology \\ 834 Chestnut Street, Suite 400 \\ Philadelphia, PA 19102 \\ Richard C. Miller, MD \\ Chairman, Department of Obstetrics \& Gynecology \\ St. Barnabas Medical Center \\ 94 Old Short Hills Road, Suite 402 \\ Livingston, NJ 07039
}

Corresponding Author:

Richard C. Miller, MD

Chairman, Department of Obstetrics \& Gynecology

St. Barnabas Medical Center

94 Old Short Hills Road, Suite 402

Livingston, NJ 07039

RMiller@sbmfm.com

Phone: 973-322-5287

Fax Number: 973-322-2309

Acknowledgements: None

Funding Sources: None

Short Title: Respiratory Issues in Ambulatory Obstetrics 


\begin{abstract}
$\underline{\text { Abstract }}$
This article reviews the diagnosis and management of the most common respiratory conditions complicating pregnancy - asthma and influenza. We also review strategies for smoking cessation in pregnancy since, in addition to exacerbating all other pulmonary conditions, smoking is the most modifiable risk factor for poor pregnancy outcome. Moreover, the obstetrician frequently encounters each of these conditions in the ambulatory setting. Thorough knowledge of the normal pregnancy-induced physiological respiratory changes combined with a comprehensive understanding of how to manage these conditions, will provide the obstetrician with the armamentarium needed to optimize health outcomes for mothers and their fetuses.
\end{abstract}

\title{
$\underline{\text { Key Words }}$
}

Asthma, Influenza, Smoking, Respiratory, Pregnancy, Ambulatory 


\section{$\underline{\text { Introduction }}$}

The diagnosis and treatment of various respiratory conditions in pregnancy can be challenging. Despite the frequency of these conditions, pregnant women may be improperly diagnosed due to failure to perform diagnostic testing or inadequately treated due to fear of medication teratogenicity. Furthermore, the pregnancy-related physiological changes of the respiratory system, designed to optimize oxygen delivery to the fetus, result in increased maternal susceptibility to respiratory insults, and may complicate the diagnostic evaluation.

This article reviews the diagnosis and management of the most common conditions affecting the respiratory system in pregnancy: asthma and influenza. Both conditions are prevalent in the Unites States and have the potential to cause significant maternal and fetal morbidity and mortality. Furthermore, a knowledgeable general obstetrician can almost always manage these conditions in pregnancy, and proper treatment improves health outcomes. Lastly, we discuss smoking in pregnancy since it is the most modifiable risk factor for poor birth outcome, ${ }^{1}$ and it increases the morbidity from all other pulmonary conditions.

\section{Pregnancy-Induced Physiological Changes of the Respiratory System}

Pregnancy induces profound changes in maternal physiology and a thorough understanding of these changes is necessary in order to properly manage the pregnant patient with respiratory disease (Table 1). Important immunologic changes also occur in pregnancy to enable survival of the fetus. In particular, decreased cell-mediated immunity potentiates the risk for infection thereby rendering pregnant women more susceptible to certain respiratory pathogens.

\section{Asthma in Pregnancy}

Asthma is the most common potentially serious respiratory problem complicating pregnancy. In the United States, 3.7 to 8.4 percent of all pregnant women are asthmatic ${ }^{2}$ and the prevalence is increasing. ${ }^{3}$ In general, asthma in pregnancy follows the rule of thirds: one-third of patients will have improvement of 
symptoms, one-third will worsen, and one-third will remain the same. ${ }^{4}$ The difficulty in managing asthma in pregnancy is that its course is unpredictable. Although most women with asthma have good pregnancy outcomes, some may experience life-threatening exacerbations requiring hospitalization. For multiparous women, asthma is expected to follow a course similar to previous pregnancies. Due to the high prevalence and unpredictable nature of asthma, the obstetrician should be familiar with its classification, evaluation, and management in pregnancy (Table 2).

\section{Pregnancy Outcomes}

Asthma can result in numerous adverse pregnancy outcomes. After adjustment for comorbidities, studies have demonstrated increased risks of preeclampsia, preterm birth, low birth weight ${ }^{5}$, diabetes mellitus, ${ }^{6}$ intrauterine growth restriction, ${ }^{6}$ congenital malformations, ${ }^{7}$ and higher rates of cesarean delivery in pregnant asthmatics. ${ }^{6 .}$ These risks appear to be higher in women with poorly controlled asthma ${ }^{8}$ suggesting that treatment may improve pregnancy outcome as well as overall maternal health. ${ }^{5}$ Some studies have shown associations between maternal use of oral corticosteroids in the first trimester and subsequent facial clefting in the fetus. ${ }^{9,10}$ However, the potential risks associated with asthma medications are lower than the risks associated with uncontrolled asthma, and it is imperative that women continue to use their asthma medications in pregnancy.

\section{Diagnosis:}

Asthma is a chronic disease of airway hyper-responsiveness and inflammation with subsequent airflow limitation. The majority of asthmatics enter pregnancy with a known diagnosis of asthma. However, diagnostic testing is warranted in patients who develop respiratory symptoms for the first time in pregnancy in the absence of a known history of asthma. The most common cause of shortness of breath in pregnancy is physiologic dyspnea but, unlike asthma exacerbations, dyspnea of pregnancy is never associated with coughing, wheezing, or chest tightness. Other potential causes of these clinical findings in pregnant women include gasteroesophageal reflux, allergic rhinitis with postnasal drip, bronchitis, pneumonia, pulmonary edema and pulmonary embolism.

In general, the diagnostic evaluation for asthma does not differ significantly in pregnancy. 
Spirometry is the foundation of the evaluation since it reliably demonstrates airflow obstruction. ${ }^{11}$ The diagnosis of asthma is confirmed by a reduced forced expiratory volume in 1 second $\left(\mathrm{FEV}_{1}\right)$ with $\geq 12$ percent improvement after inhalation of a short-acting bronchodilator as measured by spirometry, or by an increase of $\geq 10$ percent of predicted $\mathrm{FEV}_{1}$ after inhalation of a short-acting bronchodilator. ${ }^{11}$ Peak flow meters are designed for monitoring asthma control and should never be used as diagnostic tools in the office due to significant variability in their reference values for predicted peak expiratory flow measurements. In patients with normal pulmonary function, methacholine testing is often performed to confirm bronchial hyperreactivity. However, methacholine testing is not recommended in pregnancy due to lack of available safety data. ${ }^{12}$ If reversibility of airway obstruction cannot be demonstrated after beta agonist administration, yet the clinical picture is consistent with asthma, the patient should be empirically treated for presumed asthma until methacholine testing can be performed post partum. ${ }^{12}$

Most patients with asthma are sensitive to allergens, and up to 85 percent of asthmatics will have a positive skin test for one or more allergens. Skin testing for allergens is recommended for most nonpregnant patients with persistent asthma to help identify potential triggers; ${ }^{11}$ however, it is not recommended in pregnancy since it is associated with systemic reactions and anaphylaxis. As an alternative, pregnant patients with persistent asthma should undergo blood testing for IgE antibodies to specific allergens. ${ }^{12}$

\section{Classification of Asthma Severity \& Asthma Management}

Proper classification of asthma severity is critical for asthma management including initiation and adjustment of medication (Table 2). In addition to a complete physical examination, an extensive history should be elicited at the first prenatal visit including number of acute asthma exacerbations, past hospitalizations, history of intubation and mechanical ventilation, prior oral steroid use, and known environmental asthma triggers. Asthma should be classified based on self-reported asthma symptoms during a two to four week period (Table 2). It is important to recognize that the asthma classification system is dynamic and women may change classes throughout pregnancy. 
Medical management utilizes a stepwise approach to treatment in order to minimize the amount of medication necessary to control a patient's asthma. An important element of asthma management is elimination of asthma triggers such as tobacco smoke, animal dander, pollens, molds, food additives, exercise, cold weather and some medications (e.g. aspirin). Limiting or eliminating exposure to environmental triggers and allergens can significantly reduce asthma symptoms and the need for medical therapy. Some specific measures for allergen control include using mattress and pillow covers, removing pets from the home, removing carpeting, and reducing humidity to prevent mold. If exposure to allergens cannot be successfully eliminated, medications such as anti-histamines and leukotriene receptor antagonists may be useful for improved asthma control (Table 3). Similarly, if exercise is a trigger, inhalation of albuterol 5 to 60 minutes before exercise may prevent exacerbations.

Stepwise guidelines for medical management of asthma are shown in Table 2 and controller medications are shown in Table 3. Inhaled corticosteroids are the foundation for medical treatment in women with persistent asthma, and women must be counseled on the importance of using their controller medications in pregnancy. A step down in medical therapy is generally recommended for non-pregnant patients whose asthma has been well controlled for more than three months. However, since the course of asthma in pregnancy is unpredictable, it may be wise to maintain the current level of therapy in order to prevent worsening of symptoms. ${ }^{12}$ A knowledgeable obstetrician can properly manage most pregnant patients with asthma, however patients with worsening moderate asthma and those with severe asthma should also be referred to a maternal-fetal medicine specialist or pulmonologist for additional management (Table 2).

\section{Monitoring of Asthma in Pregnancy}

Both the forced expiratory volume in 1 second (FEV1) and the peak expiratory flow rate (PEFR) are reliable objective measures of airway obstruction that are unchanged by the physiological changes of pregnancy making them ideal for monitoring asthma severity. Since FEV1 can only be measured by spirometry, a handheld peak flow meter for measurement of PEFR is preferred for monitoring asthma 
control. The average PEFR in pregnancy is $380-550 \mathrm{~L} / \mathrm{min}$. A baseline personal best value should be acquired at the first prenatal visit, or preferably at a preconception visit; this value then serves as the reference from which to diagnose an exacerbation or the need for change in the medication regimen. It is helpful to then mark the patient's personal peak flow meter to identify $80 \%, 50 \%$, and less than $50 \%$ of her personal best. The goal of asthma management is to maintain PEFR measurements at greater than $80 \%$ of personal best. ${ }^{13}$ Women should be counseled to seek outpatient medical care as soon as possible when PEFR values are within the $50 \%$ to $80 \%$ range and to seek immediate emergency medical care when the PEFR is less than $50 \% .^{13}$

\section{Antepartum Surveillance \& Intrapartum Management of Asthma}

There is limited data regarding the optimal obstetrical care of patients with asthma. Women with poorly controlled asthma are at increased risk of pregnancy complications. ${ }^{5,6}$ Consequently, poorly controlled pregnant asthmatics may benefit from increased fetal surveillance in the second and third

trimesters of pregnancy. ${ }^{15}$ A common regimen is to begin weekly non-stress tests or a biophysical profile at 32 weeks' gestation with serial ultrasonography for growth assessment every 4 weeks. ${ }^{13}$ Women with uncontrolled severe asthma may require additional fetal surveillance at the discretion of the physician.

\section{Asthma Management on Labor and Delivery}

Pregnant asthmatics should continue their medication regimen as prescribed throughout labor and delivery. If systemic corticosteroids have been used in the previous 4 weeks, stress dose corticosteroids are recommended to prevent relative adrenal insufficiency (a typical regimen is hydrocortisone $100 \mathrm{mg}$ every 8 hours during labor and for 24 hours post partum). ${ }^{12,}{ }^{14}$ Lumbar anesthesia can reduce oxygen consumption in labor and may be used safely in asthmatics. ${ }^{14}$ Misoprostol can be used in pregnant asthmatics without significant risk of adverse reactions, but respiratory status should be monitored for bronchospasm. ${ }^{12}$, 14 There are no reports of bronchospasm associated with calcium channel blockers used for tocolysis or treatment of hypertension, and these medications may be used in pregnant asthmatics with proper monitoring. Magnesium sulfate has bronchodilator effects and can be used safely for seizure 
prophylaxis, tocolysis, or for fetal neuroprotection as indicated. The use of carboprost, ergonovine, and methylergonovine should be avoided since they have all been associated with bronchospasm. Similarly, indomethacin has been associated with bronchospasm in patients with aspirin sensitivity and should be avoided. 12, 14, 15

\section{Clinical Checklist}

Patient education is essential for improving compliance and self-monitoring. A significant proportion of acute asthmatic exacerbations in pregnancy have been associated with non-adherence to treatment with inhaled corticosteroids. Below is a checklist to be used by the obstetrician to help ensure patients are properly educated.

\section{Patient Education Checklist for the Obstetrician}

$\circ$ Did you counsel patient on the unpredictable course of asthma in pregnancy?

○ Did you counsel the patient to remain on asthma medications during pregnancy because one-third of patients have worsening of their asthma?

$\circ$ Did you obtain \& record a baseline PEFR measurement at the first prenatal visit?

○ Did you observe and instruct the patient on how to properly obtain PERF measurements?

$\circ$ Did you observe and instruct the patient on proper use of their inhalers?

$\circ$ Did you explain how to recognize a severe asthma exacerbation and when to seek medical attention?

○ If the patient smokes, did you strongly encourage smoking cessation? Did you refer her to a smoking cessation program and offer nicotine replacement therapy if indicated?

$\circ$ Did you identify potential triggers for asthma? Common triggers include animal dander, cockroach antigens, pollens, molds, tobacco smoke, food additives, exercise, and some medications such as aspirin and beta-blockers.

○ Have you counseled the patient on how to avoid or limit exposure to allergens?

\section{Influenza in Pregnancy}

In the United States, influenza is the eighth leading cause of death. ${ }^{16}$ Influenza infection and its related complications result in more than 36,000 deaths and 200,000 hospitalizations annually. During the 
influenza season (October to April), 5- 20 percent of the general population will become infected with the virus, ${ }^{17}$ and attack rates are even higher occur during influenza pandemics.

Influenza viruses that infect humans are classified into 3 principal types: A, B, and C. Both types A and B are associated with seasonal epidemics, but only type A viruses can cause pandemics. Minor mutations in the surface proteins of Influenza A viruses (antigenic drift) occur continuously altering the virus enough that so influenza vaccines must be updated annually. Significant changes in the surface proteins of influenza A viruses occur via mutation of avian or swine viruses resulting in the development of new human subtypes (antigenic shift); these novel influenza strains are responsible for influenza pandemics and typically result in high rates of morbidity and mortality due to the lack of immunity in the population.

\section{Pregnancy Outcomes}

The direct effects of influenza infection on the fetus are unknown. In seasonal influenza epidemics, viremia is thought to occur infrequently and vertical transmission is rare, ${ }^{18}$ though this may differ with new influenza strains. ${ }^{19}$ Although there is no evidence that influenza viruses are teratogenic, adverse pregnancy outcomes such as spontaneous abortion, stillbirth and preterm birth have been reported following both pandemic and seasonal influenza infection. ${ }^{20}$ Even if the influenza virus does not have a direct effect on the fetus, both animal and human studies suggest that significant fever may be associated with an increased risk for birth defects, especially neural tube defects. 21,22

Pregnancy is a risk factor for increased maternal morbidity and mortality from both pandemic and seasonal influenza. Unlike seasonal influenza, pandemic influenza infections typically affect younger patients rendering pregnant women even more susceptible to infection. Pregnant women with influenza infection have significantly higher rates of hospitalization compared to their non-pregnant counterparts,

and are more likely to be admitted to an intensive care unit due to severe respiratory compromise. ${ }^{23,24,25}$ The Center for Disease Control (CDC) reported that out of 347 severely ill pregnant women with H1N1 
influenza infection in 2009, there were 75 deaths and 272 admissions to intensive-care units. ${ }^{26}$ Many of these severely ill women were in the third trimester of pregnancy since disease severity increases with advancing gestational age. ${ }^{23}, 27,28$ Not surprisingly, women with underlying medical conditions are also at risk for more severe infection. During the 2009 H1N1 pandemic, asthma was the most common comorbid condition among women with severe influenza infection. ${ }^{23,29}$ Obstetricians must have a high index of suspicion for influenza in women presenting with fever and respiratory illness since infected pregnant women experience a disproportionate share of the morbidity and mortality.

\section{Diagnosis \& Testing for Influenza}

The influenza virus is spread through aerosolized respiratory droplets with symptoms typically developing one to four days after exposure. Seasonal influenza infection causes fever, chills, dry cough, headache, malaise, nausea, vomiting, rhinorrhea, and myalgia. In the 2009 H1N1 pandemic, symptoms were similar to seasonal influenza infection, but gastrointestinal symptoms such as diarrhea and vomiting were more frequent. ${ }^{28}$ Influenza infection is largely a clinical diagnosis and recommendations for laboratory tests differ between seasonal and pandemic strains. Several effective laboratory tests exist including direct antigen detection tests, viral cell culture of nasopharyngeal or throat samples, and detection of influenza-specific RNA by real-time reverse transcriptase-polymerase chain (rt-PCR) reaction. Due do the historically poor sensitivity of rapid influenza tests (ranging from 10-80\% compared to viral culture or rt-PCR), clinical decisions about treatment should not be guided or delayed by negative rapid test results. ${ }^{30}$ If influenza is prevalent in the community, women with uncomplicated illness can be diagnosed clinically, and influenza testing is not needed for antiviral treatment. Clinicians should use clinical judgment to decide when to test for influenza in women who are not severely ill since, in certain situations, diagnostic testing may help inform decisions regarding clinical care, infection control, or management of close contacts. If testing for pandemic influenza is warranted, the CDC recommends the Human Influenza Virus Real-Time PT-PCR influenza panel assay for confirmation. ${ }^{30}$

\section{Management of Influenza}


Based on the potential severity of influenza infection, pregnant women who become ill with influenza should be treated aggressively with antipyretic therapy, and should adhere to standard recommendations for folic acid consumption. ${ }^{31}$ Antiviral medications play an important role both for post-exposure prophylaxis and for treatment of infected pregnant women, particularly during influenza pandemics. The use of antiviral medications can significantly reduce maternal morbidity and mortality from influenza. Infected women who receive early anti-retroviral treatment have lower rates of intensive care unit admission and death. ${ }^{23,31}$ Therefore, antiviral treatment is recommended as soon as possible for any women with confirmed or suspected influenza regardless of gestational age. ${ }^{32}$ Anti-viral medications are most effective when started within 48 hours of the onset of symptoms, but may still have some benefit in pregnant women when administered beyond 48 hours. ${ }^{23}$ Treatment should never be delayed while awaiting diagnostic tests results. ${ }^{32}$ Pregnant women may be offered antiviral prophylaxis within 48 hours after exposure to a person with confirmed, probable, or suspected influenza infection; however, if availability of medication is an issue, public health authorities may recommend that antivirals be prescribed only for treatment. ${ }^{33}$

For the 2011-2012 influenza season, two FDA-approved neuraminidase inhibitors, zanamivir (Relenza) and oseltamivir (Tamiflu), are available for post-exposure prophylaxis and treatment. ${ }^{33}$ The prophylactic and treatment dosing regimens for these medications are listed in Table 4. Two older antiinfluenza medications, rimantidine and amantadine, are not recommended because of high rates of resistance among circulating influenza A viruses. ${ }^{32}$ Oseltamivir is preferred for both treatment and chemoprophylaxis in pregnant women since more safety data are available. To date, no teratogenic effects have been identified with either of these two medications and, considering the severity of disease, treatment benefits appear to outweigh any potential risks. ${ }^{34,35}$

Pregnant women being treated for confirmed or suspected influenza infection should be counseled on when to seek emergency medical care. Obstetricians should have a very low threshold for hospital admission in patients presenting with difficulty breathing or shortness of breath, pain or pressure 
in the chest, contractions and/or symptoms of labor, sudden dizziness, confusion, persistent vomiting, decreased or no fetal movement (in the third trimester), or a fever that is not responding to anti-pyretic therapy.

\section{Prevention}

Pregnant and postpartum women represent a vulnerable population with regard to influenza, and influenza vaccination should be an integral element of prenatal care. Historically, influenza vaccination rates in pregnancy have been unacceptably low; the highest vaccination rate was achieved in the 20092010 influenza season where $32 \%$ of pregnant women were vaccinated. ${ }^{36}$ The Advisory Committee on Immunization Practices (ACIP), the Center for Disease Control (CDC), and the American College of Obstetricians and Gynecologists (ACOG) endorse annual inactivated influenza vaccination for women who will be pregnant during the influenza season (October-mid May). Inactivated influenza vaccination is recommended in all three trimesters of pregnancy. ${ }^{37}$ While all women should be encouraged to receive the influenza vaccine, it is imperative that women with underlying respiratory and medical conditions be vaccinated since they are at even greater risk for severe disease.

Multidose vials of the vaccine contain trace thimerisol, a mercury-containing preservative, which has not been linked to neonatal, or childhood adverse outcomes. The ACIP and CDC recommend vaccination regardless of thimerosal content. Thimerosal-free vaccines are available in single-dose vials and may be used depending on patient preference. No study to date has shown any adverse consequences of influenza vaccination in pregnant women. ${ }^{38}$ Preliminary results from a trial of the 2009 H1N1 nonadjuvanted vaccine showed an immune response in pregnant women similar to the response in nonpregnant adults, and no safety concerns were identified. ${ }^{39}$ The live, attenuated influenza virus vaccine, available as an intranasal spray, is not approved for use during pregnancy given the theoretical risk associated with use of a live vaccine.

Immunity in the mother and close household contacts is the only effective strategy to prevent influenza infection in the newborn since the vaccine is not approved for use in infants less than 6 months of age. Multiple studies have demonstrated fewer cases of laboratory-confirmed influenza and lower rates 
of respiratory infections with fever among infants born to immunized mothers compared to infants of unimmunized women. ${ }^{40,41,42}$ Immunized mothers who breast feed provide their infants with maternal antibodies to influenza thereby conferring additional protection. Conversely, bottle-fed infants are more vulnerable to influenza as well as other respiratory infections, and are at increased risk for hospitalization. The risk of influenza transmission via breast milk is unknown; reports of viremia with seasonal influenza are rare which suggests that transmission of influenza into breast milk is also probably rare. Women who are ill with influenza should continue to breastfeed. In order to prevent respiratory transmission to breastfed infants, it is recommended that women with influenza-like illness use facemasks and proper hand hygiene when providing infant care and feedings. Women who are acutely ill may pump their milk for bottle feedings by a healthy family member.

\section{Smoking in Pregnancy}

A review of the diagnosis and management of respiratory conditions warrants a discussion of one of the most important public health problems in this country - smoking. Approximately $13 \%$ of pregnant women in the United States smoke ${ }^{43}$ making it one of the most preventable causes of both maternal and fetal morbidity and mortality. In addition to the known maternal health consequences, smoking is also associated with numerous adverse pregnancy and perinatal outcomes related to impaired placental gas exchange, placental insufficiency and direct toxic effects to the fetus. These adverse outcomes include placenta previa, placental abruption, preterm premature rupture of membranes, preterm birth, intrauterine growth restriction, stillbirth, low birth weight and sudden infant death syndrome. Direct toxic effects to the fetus include possible facial clefting ${ }^{44,45}$ and decreased intelligence. ${ }^{46}$ Smoking also impairs fertility, and increases the risk of ectopic pregnancy and spontaneous abortion. ${ }^{47}$ Figure 1 shows the relative excess incidence proportions of various obstetrical complications significantly associated with smoking

during pregnancy. ${ }^{48}$ Thus, decreasing the smoking rate in pregnancy may prevent a significant percentage of serious obstetrical complications. 


\section{Pregnancy: A Unique Opportunity for Smoking Cessation}

Most women seek medical care at some point during pregnancy providing the physician with a unique opportunity for smoking cessation intervention. Women are more likely to quit smoking during pregnancy than at any other time in life making pregnancy a "teachable moment" ${ }^{49,50}$ yet less than a third of obstetricians refer patients to smoking intervention programs. ${ }^{51}$ Approximately $20 \%$ of smokers will successfully abstain during pregnancy. ${ }^{52}$ Women who smoke ten or fewer cigarettes per day prior to pregnancy have quit rates double those women who smoke more than ten cigarettes per day. ${ }^{53}$ Women who successfully quit smoking in pregnancy are at very high risk of relapse in the post-partum period with relapse rates as high as $60 \%$ in the first six months, therefore it is essential that interventions continue into the postpartum period. ${ }^{49}$

\section{Benefits of Smoking Cessation in Pregnancy}

Smoking cessation in pregnancy can decrease the risk of an adverse pregnancy outcome. Women who receive smoking cessation interventions have a reduction in low birth weight infants and preterm birth, indicating that smoking cessation in pregnancy may slow or reverse its adverse effects on perinatal outcomes. ${ }^{54}$ There is evidence to suggest that women who quit smoking as late at 32 weeks' gestation deliver infants with higher birth weights compared to those who continue to smoke. ${ }^{55}$ In addition to the short-term immediate effects of smoking on pregnancy outcome, the long-term health benefits to mothers and their children must also be taken into consideration when recommending intervention strategies.

\section{Smoking Cessation Strategies}

Smoking during pregnancy is a complex and variable behavior that has many social, behavioral and physiological triggers. Smoking cessation interventions include cognitive behavioral therapy (e.g. coping skills training or behavioral contracting), interventions based on stages of change (e.g. consciousness raising techniques for patients in pre-contemplation stage), providing feedback on fetal health status or nicotine by-products measurements, and/or pharmacological treatment. ${ }^{1}$ In a large metaanalysis, trials using cognitive behavioral therapy and incentives as the main intervention strategy 
demonstrated statistically significant improvements in infant birth weight. ${ }^{1}$ There is a general consensus among experts that behavioral interventions should be encouraged before pharmacological approaches are used. Women should be asked about tobacco use and offered effective smoking cessation interventions at the first prenatal visit and again at each prenatal visit including post-partum follow-up visits. Pregnant smokers should receive augmented intervention through counseling by the obstetrician, pregnancyspecific self-help materials, and counseling sessions with a health educator. ${ }^{56}$ Brief smoking cessation counseling in conjunction with administration of pregnancy-specific self-help materials can significantly improve cessation rates compared with cessation rates achieved with just advice to quit. ${ }^{57}$ ACOG endorses the 5 As method of smoking cessation intervention adapted for use during pregnancy. ${ }^{50,56,58}$

\section{The 5 A's of Smoking Cessation:}

1. Ask the patient about tobacco use

2. Advise her to quit

3. Assess her willingness to make a quit attempt

4. Assist in her quit attempt

\section{Arrange follow-up}

Counseling alone may not significantly increase cessation rates particularly in moderate-heavy smokers since it does not address the issue of physiological dependence. ${ }^{59}$ Many smokers will experience symptoms of nicotine withdrawal, which can have a substantial negative influence on attempts at smoking cessation. ${ }^{60}$ Nicotine replacement products can help ease the symptoms of nicotine withdrawal. However, the data regarding the safety of nicotine replacement products in pregnancy are conflicting and more information is needed about their risks in pregnancy before evidence-based recommendations can be made. ${ }^{1,61,62}$ Of the possible pharmacological interventions, nicotine replacement therapy (NRT) is the treatment of choice in pregnancy in women who are moderate-heavy smokers or who are highly dependent and have been unable to quit smoking by other means. ${ }^{62,63}$ Clinicians frequently focus solely on the risk to the fetus when determining the risk-benefit ratio of pharmacological smoking cessation 
strategies. However, maternal health benefits must also be taken into consideration especially since the data indicating potential teratogenicity is limited and of poor quality. Smoking has also serious long-term health risks for infants that extend into adulthood; these risks are beyond the scope of this article but must also be considered when determining the risk-benefit ratio for pharmacological smoking cessation strategies.

Because the total amount of nicotine exposure to the fetus is less with nicotine replacement therapy than with continued moderate to heavy smoking, and because the developing fetus is not exposed to the other toxic substances found in cigarettes, transdermal nicotine patches are recommended to help with smoking cessation efforts in heavy smokers who have failed non-pharmacologic interventions. ${ }^{50}$ The goal of nicotine replacement therapy is to provide a level of nicotine just above that associated with withdrawal symptoms. The risk/benefit ratio for the use of pharmacotherapy for smoking cessation in pregnancy appears favorable in those who smoke more than 10 cigarettes per day (moderate-heavy smokers). Two randomized control trials have found that women using nicotine gum ${ }^{64}$ or the nicotine patch $^{65}$ had infants with significantly higher birth weights compared with smokers receiving placebo. Table 4 shows the available pharmacological methods for smoking cessation.

\section{Conclusion:}

Respiratory issues, particularly asthma and influenza infection, are commonly encountered by the obstetrician in the ambulatory setting; similarly, smoking is an important public health problem that is prevalent among pregnant women. Thorough knowledge of the normal pregnancy-induced physiological respiratory changes as well as a comprehensive understanding of how to manage these conditions will provide the obstetrician with the armamentarium needed to optimize outcomes for mothers and their fetuses.

\footnotetext{
${ }^{1}$ Lumley J, Oliver S, Waters E. Interventions for promoting smoking cessation during pregnancy. Cochrane Database Syst Rev 2000; 2: CD001055.
} 
${ }^{2}$ Kwon HL et al. Asthma Prevalence among Pregnant and Childbearing-aged Women in the United States: Estimates from National Health Surveys. Annals of Epidemiology 2003; 13: 317-324.

${ }^{3}$ Asthma in the US. Centers for Disease Control and Prevention Web site. http://www.cdc.gov/VitalSigns/Asthma. Updated May 4, 2011. Accessed October 30, 2011.

${ }^{4}$ Schatz M, Dombrowski MP, Wise R, et al. Asthma morbidity during pregnancy can be predicted by severity classification. J Allergy Clin Immunol 2003; 112: 283.

${ }^{5}$ Murphy et al. A meta-analysis of adverse perinatal outcomes in women with asthma. BJOG 2011; 118 : 1314-23.

${ }^{6}$ Sheiner E, Mazor M, Levy A, Wiznitzer A, Bashiri A. Pregnancy outcome of asthmatic patients: a population-based study. J Matern Fetal Neonatal Med 2005; 18: 237-40.

${ }^{7}$ Blais L, Kettani FZ, Elftouh N, Forget A. Effect of maternal asthma on the risk of specific congenital malformations: A population-based cohort study. Birth Defects Res A Clin Mol Teratol 2010; 88: 216-22.

${ }^{8}$ Bracken MD, Triche EW, Belandger K, et al. Asthma symptoms, severity, and drug therapy: a prospective study of effects on 2205 pregnancies. Obstet Gynecol 2003;102:739-52.

${ }^{9}$ Carmichael SL, Shaw GM, Ma C, Werler MM, Rasmussen SA, Lammer EJ; National Birth Defects Prevention Study. Maternal corticosteroid use and orofacial clefts. Am J Obstet Gynecol 2007; 197: 
585.e1-7.

${ }^{10}$ Rodríguez-Pinilla E, Martínez-Frías ML. Corticosteroids during pregnancy and oral clefts: a casecontrol study. Teratology 1998; 58: 2-5.

${ }^{11}$ National Asthma Education and Prevention Program. Expert panel report 3: guidelines for the diagnosis and management of asthma: full report 2007. (Accessed October 5, 2011, at http://www.nhlbi.nih. gov/guidelines/asthma/asthgdln.pdf.)

${ }^{12}$ Schatz M. Dombrowski MP. Clinical Practice. Asthma in Pregnancy. NEJM 2009; 360: 1862-9.

${ }^{13}$ National Asthma Education and Prevention Program: Full Report of the Expert Panel: Guidelines for the Diagnosis and Management of Asthma, 2007. Available at http://www.nhlbi.nih.gov/health/prof/lung/asthma/astpreg/astpreg_full.pdf (Accessed November 2011)

${ }^{14}$ Hardy-Fairbanks AJ, Baker ER. Asthma in pregnancy: pathophysiology, diagnosis and management. Obstet Gynecol Clin North Am 2010 Jun; 37: 159-72.

${ }^{15}$ Dombrowski MP, Schatz M; ACOG Committee on Practice Bulletins-Obstetrics. ACOG practice bulletin: clinical management guidelines for obstetrician-gynecologists number 90, February 2008: asthma in pregnancy. Obstet Gynecol 2008 Feb; 111: 457-64. 
${ }^{16}$ Heron M, Hoyert D, Murphy S, Xu J, Kochanek K, Tejada-Vera B. Deaths: Final Data for 2006. National vital statistics reports; vol 57, no 14. Hyattsville, MD: National Center for Health Statistics. 2009.

${ }^{17}$ Seasonal Influenza: Questions and Answers. Centers for Disease Control and Prevention Web site. http://www.cdc.gov/flu/about/qa/disease.htm. Updated July 6, 2011. Accessed November 3, 2011.

${ }^{18}$ Irving WL et al. Influenza virus infection in the second and third trimesters of pregnancy: a clinical and seroepidemiological study. BJOG 2000; 107: 1282-9.

${ }^{19}$ Xu L, Bao L, Deng W, Qin C. Highly pathogenic avian influenza H5N1 virus could partly be evacuated by pregnant BALB/c mouse during abortion or preterm delivery. Virol J 2011; 8: 342.

${ }^{20}$ Pierce M et al. Perinatal outcomes after maternal 2009/H1N1 infection: national cohort study. BMJ 2011; 342: d3214

${ }^{21}$ Moretti ME, Bar-Oz B, Fried S, Koren G. Maternal hyperthermia and the risk for neural tube defects in offspring: systematic review and meta-analysis. Epidemiology 2005; 16: 216-9.

${ }^{22}$ Acs N, Bánhidy F, Puhó E, Czeizel AE. Maternal influenza during pregnancy and risk of congenital abnormalities in offspring. Birth Defects Res A Clin Mol Teratol 2005; 73 :989-96.

${ }^{23}$ Siston et al. Pandemic 2009 influenza A (H1N1) virus illness among pregnant women in the United States. JAMA 2010; 303: 1517-25. 
${ }^{24}$ Neuzil et al. Impact of influenza on acute cardiopulmonary hospitalizations in pregnant women. Am J Epidemiol 1998; 148: 1094-102.

${ }^{25}$ Jamieson D et al. H1N1 2009 influenza virus infection during pregnancy in the USA. Lancet 2009; 374: $451-8$

${ }^{26}$ CDC. Maternal and infant outcomes among severely ill pregnant and postpartum women with 2009 pandemic influenza A (H1N1)--United States, April 2009-August 2010. MMWR; 2011; 60:1193-6.

${ }^{27}$ Creanga AA et al. Seasonal and 2009 pandemic influenza A (H1N1) virus infection during pregnancy: a population-based study of hospitalized cases. Am J Obstet Gynecol 2011; 204: S38-45.

${ }^{28}$ Louie JK, Acosta M, Jamieson DJ, Honein MA; Severe 2009 H1N1 influenza in pregnant and postpartum women in California. NEJM 2010; 362: 27-35

${ }^{29}$ Miller AC, Safi F, Hussain S, Subramanian RA, Elamin EM, Sinert R. Novel influenza A(H1N1) virus among gravid admissions. Arch Intern Med 2010; 170: 868-73.

${ }^{30}$ Guidance for Clinicians on the Use of Rapid Influenza Diagnostic Tests. Centers for Disease Control and Prevention. Web site: http://www.cdc.gov/flu/professionals/diagnosis/clinician_guidance_ridt.htm. Updated September 30, 2011. Accessed November 15, 2011. 
${ }^{31}$ Acs N, Bánhidy F, Puhó E, Czeizel AE. Maternal influenza during pregnancy and risk of congenital abnormalities in offspring. Birth Defects Res A Clin Mol Teratol 2005; 73: 989-96.

${ }^{32}$ Fiore AE, Fry A, Shay D, Gubareva L, Bresee JS, Uyeki TM; Centers for Disease Control and Prevention (CDC). Antiviral agents for the treatment and chemoprophylaxis of influenza --recommendations of the Advisory Committee on Immunization Practices (ACIP). MMWR 2011; 60: 1-24

${ }^{33}$ 2011-2012 Influenza Antiviral Medications: A Summary for Clinicians. Centers for Disease Control and Prevention. Web site: http://www.cdc.gov/flu/professionals/antivirals/summary-clinicians.htm. Updated October 5, 2011. Accessed November 27, 2011.

${ }^{34}$ Tanaka et al. Safety of neuraminidase inhibitors against novel influenza A (H1N1) in pregnant and breastfeeding women. CMAJ 2009; 181: 55-8

${ }^{35}$ Greer LG, Sheffield JS, Rogers VL, et al. Maternal and neonatal outcomes after antepartum treatment of influenza with antiviral medications. Obstet Gynecol 2010; 115: 711-6.

${ }^{36}$ CDC. Influenza vaccination coverage among pregnant women---United States, 2010--11 influenza season. MMWR 2011;60:1078--82.

${ }^{37}$ American College of Obstetricians and Gynecologists Committee No. 468. Influenza Vaccination During Pregnancy. American College of Obstetricians and Gynecologists Committee on Obstetric Practice. Obstet Gynecol. 2010 Oct;116(4):1006-7. 
${ }^{38}$ Tamma PD, Ault KA, del Rio C, Steinhoff MC, Halsey NA, Omar SB. Safety of influenza vaccination during pregnancy. Am J Obstet Gynecol 2009; 201: 547-52.

${ }^{39}$ Interim results: influenza A (H1N1) 2009 monovalent vaccination coverage-United States, OctoberDecember 2009. Centers for Disease Control and Prevention (CDC). MMWR 2010; 59:44-8.

${ }^{40}$ Zaman K, Roy E, Arifeen SE, Rahman M, Raqib R, Wilson E, et al. Effectiveness of maternal influenza immunization in mothers and infants. NEJM 2008; 359: 1555-64.

${ }^{41}$ Benowitz et al. Influenza Vaccine Given to Pregnant Women Reduces Hospitalization Due to Influenza in Their Infants. Clinical Infectious Diseases 2010; 51:1355-1361

${ }^{42}$ Poehling KA. Impact of maternal immunization on influenza hospitalizations in infants. Am J Obstet Gynecol 2011; 204: S141-8.

${ }^{43}$ Tong VT, Jones JR, Dietz PM, D’Angelo D, Bombard JM. Trends in smoking before, during, and after pregnancy - Pregnancy Risk Assessment Monitoring System (PRAMS), United States, 31 sites, 20002005. MMWR 2009; 58: 1-29.

${ }^{44}$ Chung KC, Kowalski CP, Kiim HY, Buchman SR. Maternal cigarette smoking during pregnancy and the risk of having a child with cleft lip/palate. Plast Reconstr Surg 2000;105:485-91.

${ }^{45}$ Morales-Suarez-Varela MM, Bille C, Christensen K, Olsen J. Smoking habits, nicotine use, and congenital malformations. Obstet Gynecol 2006;107:51-57. 
${ }^{46}$ Fried PA, Watkinson B, Gray R. Differential effects on cognitive functioning in 13- to 16-year-olds prenatally exposed to cigarettes and marihuana. Neurotoxicol Teratol. 2003 Jul-Aug;25(4):427-436.

${ }^{47}$ U.S. Department of Health and Human Services. The Health Consequences of Smoking: A Report of the Surgeon General. U.S. Department of Health and Human Services, Public Health Service, Office of the Surgeon General, 2004.

${ }^{48}$ Hayashi et al, Smoking During Pregnancy Increases Risks of Various Obstetric Complications: A Case-Cohort Study of the Japan Perinatal Registry Network Database. J Epidemiol 2011; 21: 61-66.

${ }^{49}$ McBride CM, Emmons KM, Lipkus IM. Understanding the potential of teachable moments: the case of smoking cessation. Health Educ. Res 2003;18: 156-170.

${ }^{50}$ American College of Obstetricians and Gynecologists. Smoking cessation during pregnancy (Committee Opinion No. 471). Obstet \& Gynecol 2010; 116: 1241-1244.

${ }^{51}$ Jordan TR, Dake JR, Price JH. Best practices for smoking cessation in pregnancy: do obstetrician/gynecologists use them in practice? J Womens Health 2006; 15: 400-41.

${ }^{52}$ Kim SY, England LJ, Kendrick JS, Dietz PM, Callaghan WM. Am J Public Health 2009; 99: 893-8.

${ }^{53}$ Colman GJ, Joyce T. Trends in smoking before, during, and after pregnancy in ten states. American Journal of Preventive Medicine 2003; 24: 29-35. 
${ }^{54}$ Lumley J, Chamberlain C, Dowswell T, Oliver S, Oakley L, Watson L. Interventions for promoting smoking cessation during pregnancy (review). Cochrane Database Syst Rev 2009; 3.CD001055.

${ }^{55}$ Lindley AA, Becker S, Gray RH, Herman AA. Effect of continuing or stopping smoking during pregnancy on infant birth weight, crown-heel length, head circumference, ponderal index, and brain:body weight ratio. Am J Epidemiol 2000; 152: 219-25.

${ }^{56}$ Fiore et al. (2008). Treating Tobacco Use and Dependence: 2008 Update. Clinical Practice Guideline. Rockville, MD: USDHHS, PHS.

${ }^{57}$ Melvin CL, Dolan-Mullen P, Windsor RA, Whiteside HP Jr, Goldenberg RL. Recommended cessation counseling for pregnant women who smoke: A review of the evidence. Tobacco Control 2000; 9: III80.

${ }^{58}$ Chapin J, Root W. Improving obstetrician-gynecologist implementation of smoking cessation guidelines for pregnant women: An interim report of the American College of Obstetricians and Gynecologists. Nicotine Tobacco Res 2004; 6: S253.

${ }^{59}$ Filion K et al. The effect of smoking cessation counselling in pregnant women: a meta-analysis of randomised controlled trials. BJOG 2011; 118: 1422-1428

${ }^{60}$ Glynn DA, Cryan JF, Kent P, Flynn RA, Kennedy MP. Update on smoking cessation therapies. Adv Ther 2009; 26: 369-82.

${ }^{61}$ Counseling and interventions to prevent tobacco use and tobacco-caused disease in adults and pregnant women: U.S. Preventive Services Task Force reaffirmation recommendation statement. U.S. Preventive Services Task Force. Ann Intern Med 2009; 150: 551-5.

${ }^{62}$ Osadchy A Kazmin A, Koren G. Nicotine replacement therapy during pregnancy: recommended or not 
recommended? J Obstet Gynaecol Can 2009; 31: 744-7.

${ }^{63}$ Clark SM and Nakad R. Pharmacotherapeutic Management of Nicotine Dependence in Pregnancy. Obstet Gynecol Clin N Am 2011; 38: 297-311

${ }^{64}$ Oncken C, Dornelas E, Greene J, Sankey H, Glasmann A, Feinn R, Kranzler HR. Nicotine gum for pregnant smokers: a randomized controlled trial. Obstet Gynecol 2008; 112: 859-67.

${ }^{65}$ Wisborg K, Henriksen TB, Jespersen LB, Secher NJ. Nicotine patches for pregnant smokers: a randomized controlled study. Obstet Gynecol 2000; 96: 967-71.

\section{Figure 1 Legend}

Figure 1. Age Adjusted Relative Excess Incidence Proportion (REI) of Obstetric Complications Associated with Smoking During Pregnancy.

Relative excess incidence proportions (REIs) are identical to attributable fractions in the exposed population. The REI is the fraction of the obstetric complication burden among smokers during pregnancy that would not have occurred if the smokers had the same incidence of complications as nonsmokers during pregnancy.

Hayashi et al. Smoking During Pregnancy Increases Risks of Various Obstetric Complications: A Case-Cohort Study of the Japan Perinatal Registry Network Database. J Epidemiol 2011; 21: 61-66. 